2013

\title{
Quasi-Steady Stages In The Process Of Premixed Flame Acceleration In Narrow Channels
}

D. M. Valiev

V. Bychkov

V.Akkerman

L.-E. Eriksson

C. K. Law

Follow this and additional works at: https://researchrepository.wvu.edu/faculty_publications

\section{Digital Commons Citation}

Valiev, D. M.; Bychkov, V.; Akkerman, V.; Eriksson, L.-E.; and Law, C. K., "Quasi-Steady Stages In The Process Of Premixed Flame Acceleration In Narrow Channels" (2013). Faculty Scholarship. 648.

https://researchrepository.wvu.edu/faculty_publications/648 


\title{
Quasi-steady stages in the process of premixed flame acceleration in narrow channels
}

\author{
D. M. Valiev, ${ }^{1}$ V. Bychkov, ${ }^{2}$ V. Akkerman, ${ }^{1,3}$ L.-E. Eriksson, ${ }^{4}$ and C. K. Law ${ }^{1,5}$ \\ ${ }^{1}$ Department of Mechanical and Aerospace Engineering, Princeton University, \\ Princeton, New Jersey 08544-5263, USA \\ ${ }^{2}$ Department of Physics, Umeå University, SE-901 87 Umeå, Sweden \\ ${ }^{3}$ Department of Mechanical and Aerospace Engineering, West Virginia University, \\ Morgantown, West Virginia 26506-6106, USA \\ ${ }^{4}$ Department of Applied Mechanics, Chalmers University of Technology, \\ 41296 Gothenburg, Sweden \\ ${ }^{5}$ Center for Combustion Energy, Tsinghua University, Beijing 100084, China
}

(Received 21 January 2013; accepted 9 August 2013; published online 6 September 2013)

The present paper addresses the phenomenon of spontaneous acceleration of a premixed flame front propagating in micro-channels, with subsequent deflagration-todetonation transition. It has recently been shown experimentally [M. Wu, M. Burke, S. Son, and R. Yetter, Proc. Combust. Inst. 31, 2429 (2007)], computationally [D. Valiev, V. Bychkov, V. Akkerman, and L.-E. Eriksson, Phys. Rev. E 80, 036317 (2009)], and analytically [V. Bychkov, V. Akkerman, D. Valiev, and C. K. Law, Phys. Rev. E 81, 026309 (2010)] that the flame acceleration undergoes different stages, from an initial exponential regime to quasi-steady fast deflagration with saturated velocity. The present work focuses on the final saturation stages in the process of flame acceleration, when the flame propagates with supersonic velocity with respect to the channel walls. It is shown that an intermediate stage may occur during acceleration with quasi-steady velocity, noticeably below the Chapman-Jouguet deflagration speed. The intermediate stage is followed by additional flame acceleration and subsequent saturation to the Chapman-Jouguet deflagration regime. We elucidate the intermediate stage by the joint effect of gas pre-compression ahead of the flame front and the hydraulic resistance. The additional acceleration is related to viscous heating at the channel walls, being of key importance at the final stages. The possibility of explosion triggering is also demonstrated. () 2013 AIP Publishing LLC. [http://dx.doi.org/10.1063/1.4819885]

\section{INTRODUCTION}

In the process of deflagration-to-detonation transition (DDT) a (deflagration) flame front could accelerate spontaneously with a velocity increase by up to 3 orders of magnitude. This process could then trigger an explosion in the fresh fuel mixture, which subsequently evolves into detonation. ${ }^{1-8}$ The phenomena of flame acceleration and DDT play the key role in safety issues, ${ }^{3,5,6}$ pulse-detonation engines, ${ }^{4,5}$ astrophysical events, ${ }^{9,10}$ and advanced materials. ${ }^{11-13}$ A common configuration for DDT studies is that of an accelerating flame front propagating in a tube/channel from a closed end to an open one.

The first qualitative explanation of the flame acceleration was suggested by Shelkin more than 70 years ago, ${ }^{1}$ with the basic idea involving a positive feedback between expansion of the burned gas and a strongly non-uniform profile of the gas velocity due to the non-slip boundary conditions at the wall. Since then DDT was observed in numerous experiments, ${ }^{3-6,14-17}$ although, there was little progress in a quantitative theoretical understanding of the process because of complications related to turbulent burning. Only recently, starting with the notion of laminar DDT, the qualitative Shelkin mechanism has been developed into a quantitative analytical theory, ${ }^{18,19}$ having been validated through extensive numerical simulations. Additional effects of hydraulic resistance, gas retardation 
at the rough walls, and viscous drag in relation to DDT have been also discussed. ${ }^{20-23}$ An important theoretical prediction of Refs. 18 and 19 was the fast flame front acceleration in narrow channels. Following this prediction, experiments on DDT in micro-channels with diameters about $1 \mathrm{~mm}$ have been performed ${ }^{24-27}$ using ethylene-oxygen mixtures. In addition to supporting the main theoretical predictions, the experiments also demonstrated several additional features beyond the scope of the theory, such as the possibility of fast steady or quasi-steady deflagration propagating with supersonic speed with respect to the channel wall. Since the theory ${ }^{18,19}$ was restricted to the initial incompressible stage of flame acceleration, it did not account for compressibility effects.

To clarify the transition from the initial to final stages of the flame acceleration process, an extensive numerical study was subsequently undertaken ${ }^{28}$ covering three orders of magnitude variation in the flame front velocity, with the flame accelerating from a realistically small initial velocity (corresponding to the initial Mach number $M a \sim 10^{-3}$ ) to supersonic speed in the laboratory reference frame. It was shown that the flame acceleration undergoes three distinctive stages: (1) initial exponential acceleration in a quasi-isobaric regime in agreement with Refs. 18 and 19; (2) almost linear increase in the flame speed to supersonic values; and (3) saturation to a quasi-steady high-speed deflagration velocity, which is eventually followed by explosion of the fuel mixture and consequently DDT. The role of gas compressibility in moderating flame acceleration in smooth channels has been also demonstrated analytically, with the assumption of a small but finite Mach number, in Ref. 29. When analyzing the numerical results of Ref. 28 related to the end of the acceleration and prior to detonation initiation, one should naturally expect saturation of the deflagration speed to the limiting Chapman-Jouguet $(\mathrm{CJ})$ value, $U_{\mathrm{CJ}}$, predicted by the classical theory. ${ }^{30,31}$ Surprisingly, the saturation velocity obtained in Ref. 28 was noticeably smaller than the CJ deflagration speed, being $\sim 0.6 U_{\mathrm{CJ}}$. Experimental results ${ }^{24-27}$ also demonstrated the possibility of a lower saturation velocity as compared to the $\mathrm{CJ}$ deflagration speed, thus supporting the numerical prediction. Moreover, results from a number of studies showed step-wise flame acceleration in the final stages, ${ }^{32,33}$ with an intermediate "plateau" in the evolution of the flame front velocity, which is followed by additional acceleration. Since such a step-wise flame acceleration would lead to generation of pressure/shock waves by the flame, fuel pre-heating and, consequently, DDT run-up distance, all these problems require further study of the intermediate quasi-steady stages of flame acceleration.

The goal of the present work is to study computationally the final stages in the process of flame acceleration and DDT in smooth channels. Focusing mainly on the final stages, we consider relatively large initial values of the Mach number, which implies smaller maximum distance between the flame front and the leading shock wave, thus reducing solution size and allowing for more comprehensive parametric study as compared to Ref. 28. In contrast to the previous numerical studies, here we show that saturation of the flame speed to the CJ deflagration velocity value occurs in two steps. First, we obtain an intermediate quasi-steady saturation to values noticeably lower than the CJ deflagration speed in agreement with the previous numerical results. ${ }^{28}$ We demonstrate that the intermediate quasi-steady saturation regime is related to gas pre-compression and viscous stress at the channel walls. We theoretically analyze the possibility of the intermediate quasi-steady saturation as the result of gas pre-compression by the accelerating flame. The analysis accounts for strong dependence of the acceleration rate on the thermal expansion ratio at the flame front, so that the acceleration stops completely below a certain critical value of the expansion ratio. ${ }^{18,19,34}$ Second, after the intermediate quasi-stationary regime, an additional acceleration of the flame front is observed with subsequent saturation to the CJ deflagration regime. We emphasize that both the first and the second saturation regimes develop prior to explosion of the fuel mixture and detonation initiation. We also demonstrate the possibility of explosion and detonation triggering. The explosion develops in an essentially multidimensional manner, with fast spontaneous reaction spreading along the walls, pushing rather strong shocks and producing powerful hydrodynamic instabilities.

\section{THEORETICAL MODEL OF THE QUASI-STEADY FLAME TIP VELOCITY}

The intermediate quasi-stationary stage of the flame acceleration is a result of the coupling of several mechanisms. In this section, we evaluate the role of one mechanism, namely, a strong reduction of the expansion ratio with flame acceleration. The other factor is the hydraulic resistance 


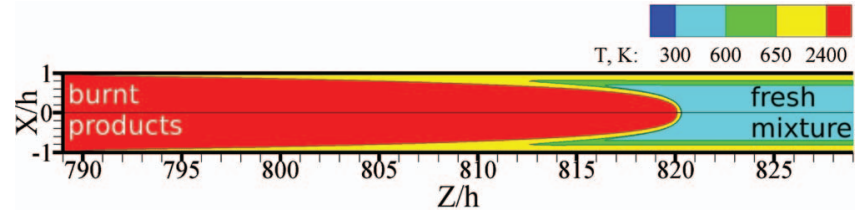

FIG. 1. A flame shape in a channel with non-slip at the walls. $R e=13.3$, scaled time instant $S_{L} t / h=6.69$; mark squeezing of the figure along the $\mathrm{z}$-axis is made for illustrative purposes.

due to viscous stress leading to momentum and energy loss, which was studied extensively in Refs. 20 and 21 and references therein.

We consider a flame front propagating in a 2D, planar semi-infinite channel (i.e., with one end closed) of half-width $h$, with non-slip adiabatic walls, as illustrated in Fig. 1. According to the incompressible analysis of Refs. 18 and 19, with further development in Ref. 34, flames in tubes/channels can accelerate only if the thermal expansion ratio $\Theta$, defined as unburnt-to-burnt density ratio $\rho_{u} / \rho_{b}$ (equal to the burnt-to-unburnt temperature ratio $T_{b} / T_{u}$ for small laminar flame speeds), exceeds a certain critical value, being $\Theta_{C}=3$ for the planar semi-infinite channel under consideration. At the initial stage of this acceleration, the flame-generated flow is almost incompressible, and the acceleration is exponential ${ }^{18}$

$$
U_{w} / S_{L} \propto \exp \left(\sigma S_{L} t / h\right),
$$

where $U_{w}$ is the total burning rate and $S_{L}$ is the unstretched laminar flame speed. In the limit of strong acceleration associated with the large thermal expansion $\Theta \gg 1$, the acceleration rate may be calculated using a simple analytical formula ${ }^{18}$

$$
\sigma=\frac{(\operatorname{Re}-1)^{2}}{4 \operatorname{Re}}\left[\sqrt{1+\frac{4 \operatorname{Re} \Theta}{(\operatorname{Re}-1)^{2}}}-1\right]^{2},
$$

where $R e=R S_{L} / v$ is the Reynolds number related to the flame propagation and $v$ is the kinematic viscosity. However, for the present work the limit of relatively weak acceleration rate is most relevant. In that case, the acceleration rate has to be found numerically from the equation ${ }^{18}$

$\frac{\sqrt{\sigma R e} \cosh \sqrt{\sigma R e}-\sinh \sqrt{\sigma R e}}{\sqrt{\sigma R e}(\Theta-1)}=\frac{\exp \sqrt{\sigma R e}}{2(\sqrt{\sigma R e}+\sigma)}-\frac{\exp (-\sqrt{\sigma R e})}{2(\sqrt{\sigma R e}-\sigma)}+\frac{\sigma R e}{\sigma R e-\sigma^{2}} \frac{\exp (-\sigma)}{\sigma}-\frac{1}{\sigma}$.

Figure 2 presents the dependence of the acceleration rate on the expansion factor found as a numerical solution to Eq. (3). We stress that the numerical solution predicts the existence of a minimum value of the thermal expansion ratio $\Theta$, at which acceleration is possible. Such a limiting value is missing in the simplified analytical formula of $\sigma$ given by Eq. (2). Though the accelerating flame is strongly curved, at sufficiently large scales its propagation may be treated as a motion of a quasi-1D, semitransparent piston, ${ }^{35}$ rendering the entire problem to be quasi-1D as well.

While compressibility and viscous heating are of minor importance at the initial stage of the process, they subsequently come to play with the flame acceleration, as the accelerating flame front generates compression/shock waves in the fuel mixture. The compression waves reduce the instantaneous mean thermal expansion ratio $\theta$ at the flame front as compared to its initial value $\theta_{0}=\Theta$, see Ref. 29, thereby reducing the acceleration rate $\sigma$ as well, see Eq. (2). Eventually, $\theta$ approaches the critical value, $\theta=\Theta_{C}=3$, which reduces the acceleration rate strongly and may result in the first quasi-steady stage, with a fast, near-sonic deflagration speed. It is noted, however, that such a primary, quasi-steady "plateau" on the velocity evolution is limited in time, because viscous heating in the transverse (radial) direction becomes dominant at a certain stage, ${ }^{33}$ hence making the quasi-1D, self-similar formulation invalid along with cessation of the initial quasi-steady plateau in the velocity evolution. Consequently, the flame front accelerates again and approaches a secondary plateau, which corresponds to the CJ deflagration velocity and lasts until the explosion onset. 


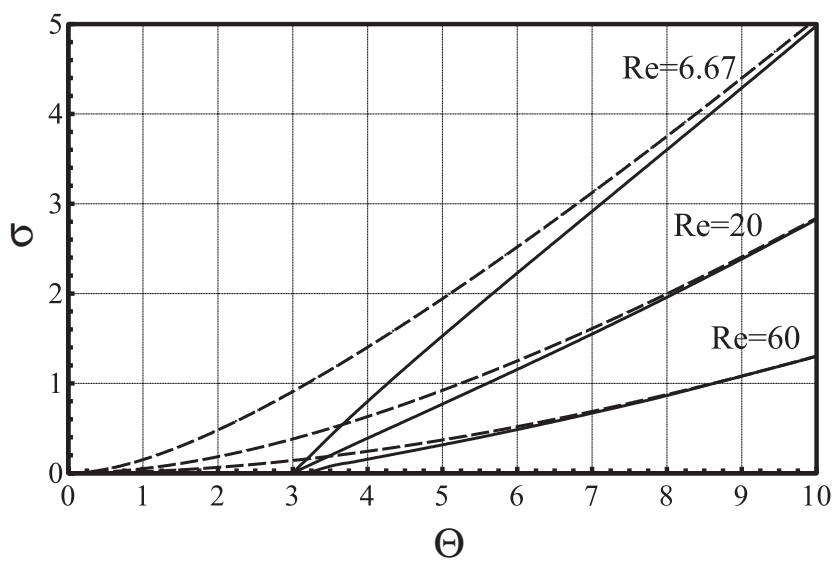

FIG. 2. Dependence of the acceleration rate on the expansion ratio according to theoretical analysis. ${ }^{18}$ Solid line: Eq. (3), dashed line: Eq. (2) obtained within limit of large thermal expansion.

To develop a quantitative model for this mechanism, we first neglect viscous heating, focusing on gas compression, and assume the flow to be isentropic, which holds with a good accuracy at the initial stages of flame acceleration. The instantaneous scaled temperature increase ahead of the flame is ${ }^{35}$

$$
\vartheta_{\text {comp }}=\frac{T}{T_{u}}=\left[1+\frac{\gamma-1}{2}(\Theta-1) M a_{w}\right]^{2},
$$

where $M a_{w}=U_{w} / c_{s}$ is the instantaneous Mach number related to the flame propagation with respect to the unburnt gas, $c_{s}$ is the initial sound speed in the fuel mixture, and $\gamma=C_{p} / C_{v}$ is the adiabatic index. The flame acceleration stops if the instantaneous temperature ratio becomes as low as the critical value:

$$
\Theta_{C}=T_{b} / T=\frac{\Theta}{\left[1+\frac{\gamma-1}{2}(\Theta-1) M a_{w}\right]^{2}},
$$

which yields

$$
M a_{w}=2 \frac{\sqrt{\Theta / \Theta_{C}}-1}{(\gamma-1)(\Theta-1)},
$$

with the saturation Mach number for the flame tip in the laboratory frame $M a_{\text {tip }}$ being

$$
M a_{\text {tip }}=\Theta_{C} M a_{w}=2 \Theta_{C} \frac{\sqrt{\Theta / \Theta_{C}}-1}{(\gamma-1)(\Theta-1)} .
$$

In particular, for $\Theta=8, \Theta_{C}=3$, and $\gamma=1.4$, the model predicts intermediate saturation for $M a_{w} \approx 0.45$ and $M a_{\text {tip }}=\Theta_{C} M a_{w} \approx 1.35$. It is noted that the saturation of the flame velocity in Ref. 28 has been obtained for $M a_{\text {tip }} \approx 2.0$ and the same initial expansion ratio $\Theta=8$, with the CJ deflagration speed corresponding to $M a_{\mathrm{CJ}, \text { tip }} \approx 2.7$.

It is noted that the above estimations are viable only for large Reynolds numbers, i.e., for sufficiently wide channels with negligible viscous heating at the channel wall. For narrower channels, we have to modify the model incorporating viscous heating in the instantaneous average temperature rise ahead of the flame in the form

$$
\frac{T}{T_{u}}=\left(1+\left\langle\vartheta_{\text {visc }}\right\rangle\right) \vartheta_{\text {comp }},
$$


where $\left\langle\vartheta_{\text {visc }}\right\rangle$ denotes contribution to the gas pre-heating due to viscous stress averaged over the channel width. The scaled temperature profile near the channel wall is given by ${ }^{33}$

$$
\vartheta(\xi)=\frac{(\gamma-1) \operatorname{Pr}(\Theta-1)^{2} \eta^{2}}{2(2-\operatorname{Pr})(\eta-1)^{2}} M a_{w}{ }^{2}\left[\sqrt{\frac{2}{\operatorname{Pr}}} \exp \{\sqrt{2 \operatorname{Pr}} \eta(\xi-1)\}-\exp \{2 \eta(\xi-1)\}\right],
$$

which can be integrated over the channel width as

$$
\left\langle\vartheta_{\text {visc }}\right\rangle=\int_{0}^{1} \vartheta(\xi) d(\xi)=\frac{(\gamma-1) \operatorname{Pr}(\Theta-1)^{2} \eta^{2}}{2(2-\operatorname{Pr})(\eta-1)^{2}} \psi M a_{w}^{2},
$$

with an auxiliary factor

$$
\psi=\frac{1-\exp (-\sqrt{2 \operatorname{Pr}} \eta)}{\operatorname{Pr} \eta}-\frac{1-\exp (-2 \eta)}{2 \eta} .
$$

Here, $\xi=x / h$ is the scaled coordinate normal to the channel wall, $\eta=\sqrt{\sigma R e}$ characterizes the curvature of the flame shape, and $P r$ is the Prandtl number of the unburnt gas. Substituting Eqs. (4), (9), and (10) into Eq. (7), taking into account that $T / T_{u}=\Theta / \Theta_{C}$ in the steady regime (with $\Theta_{C}=3$ ), and dropping terms of the third and higher orders of $M a_{w}$, we obtain an equation for the steady value of $M a_{w}$ :

$$
\begin{array}{r}
M a_{w}^{2}\left[\frac{(\gamma-1)^{2}(\Theta-1)^{2}}{4}+\frac{(\gamma-1) \operatorname{Pr}(\Theta-1)^{2} \eta^{2}}{2(2-\operatorname{Pr})(\eta-1)^{2}} \psi\right] \\
+M a_{w}(\gamma-1)(\Theta-1)+\left(1-\Theta / \Theta_{C}\right)=0 .
\end{array}
$$

For $\Theta=8-14, R e=0-50$, relevant to our studies, Eq. (11) has one positive and one negative root, with the former being

$$
M a_{w}=-\frac{(\gamma-1)(\Theta-1)}{2 A}+\sqrt{\frac{(\gamma-1)^{2}(\Theta-1)^{2}}{4 A^{2}}-\frac{1-\Theta / \Theta_{C}}{A}},
$$

where

$$
A=\frac{(\gamma-1)^{2}(\Theta-1)^{2}}{4}+\frac{(\gamma-1) \operatorname{Pr}(\Theta-1)^{2} \eta^{2}}{2(2-\operatorname{Pr})(\eta-1)^{2}} \psi .
$$

Consequently, the Mach number relevant to the flame tip in the laboratory frame is

$$
M a_{\text {tip }}=\Theta_{C} M a_{w}=-\frac{\Theta_{C}(\gamma-1)(\Theta-1)}{2 A}+\Theta_{C} \sqrt{\frac{(\gamma-1)^{2}(\Theta-1)^{2}}{4 A^{2}}-\frac{1-\Theta / \Theta_{C}}{A}} .
$$

Generally speaking, the narrower is the channel, the greater is the contribution of viscous heating to the instantaneous expansion ratio. For the range of $R e$ employed in the present numerical simulations, viscous heating produces only a minor modification of the instantaneous expansion ratio at the initial acceleration stage, and a relatively small modification to the saturation value of $M a_{\text {tip }}$, see Sec. IV for details. It is expected that the relative contribution of the viscous heating at the wall should be larger in cylindrical configuration as compared to planar geometry. It is noted that the above model is based on the "critical density ratio" concept of Ref. 34 for the hypothetical steady flame propagation, which employs the assumption of an established Poiseuille flow in the channel, while in reality the Poiseuille velocity profile may not fully develop as the critical expansion ratio is attained. It is also noted that Eq. (8) is derived in the low-Mach limit ${ }^{33}$ and assumes exponential flame acceleration, which is no longer valid at the very end of flame acceleration and at the saturation stage; the subsequent process is discussed in Sec. IV. For all these reasons, the present analysis should be treated as an assessment rather than a rigorous theory. As we will see from the numerical results, the compression waves do reduce the thermal expansion at the flame considerably, from $\theta_{0}=\Theta=8$ to $\theta=5$, and the deviation from the critical value drops even stronger, from $\Theta-\Theta_{C}=5$ to $\theta-\Theta_{C} \approx 2$. Nevertheless, the reduction is not as strong as required by the theoretical model for the saturation. Therefore, in order to explain the intermediate quasi-saturation 
regime one should look for additional mechanisms that weaken the acceleration. In this sense, another possible candidate is the hydraulic resistance, considered, e.g., in Ref. 21.

\section{BASIC EQUATIONS AND NUMERICAL APPROACH}

We perform numerical simulation of the 2D Cartesian hydrodynamic and combustion equations including transport and chemical kinetics. In tensor form the governing equations are

$$
\begin{gathered}
\frac{\partial}{\partial t} \rho+\frac{\partial}{\partial x_{i}}\left(\rho u_{i}\right)=0, \\
\frac{\partial}{\partial t}\left(\rho u_{i}\right)+\frac{\partial}{\partial x_{j}}\left(\rho u_{i} u_{j}+\delta_{i j} P-\tau_{i j}\right)=0, \\
\frac{\partial}{\partial t}\left(\rho \varepsilon+\frac{1}{2} \rho u_{i} u_{j}\right)+\frac{\partial}{\partial x_{i}}\left(\rho u_{i} \bar{h}+\frac{1}{2} \rho u_{i} u_{j} u_{j}+q_{i}-u_{j} \tau_{i j}\right)=0, \\
\frac{\partial}{\partial t}(\rho Y)+\frac{\partial}{\partial x_{i}}\left(\rho u_{i} Y-\frac{\mu}{S c} \frac{\partial Y}{\partial x_{i}}\right)=-\frac{\rho Y}{t_{R}} \exp \left(-E_{a} / R^{0} T\right),
\end{gathered}
$$

where $Y$ is the fuel mass fraction, $\varepsilon=Q Y+C_{v} T$ is the internal energy, $\bar{h}=Q Y+C_{p} T$ is the enthalpy, $Q$ is the chemical energy release, and $C_{v}$ and $C_{p}$ are the heat capacities at constant volume and pressure, respectively. $\delta_{i j}$ is the Kronecker delta. The stress tensor $\tau_{i j}$ and the energy diffusion vector $q_{i}$ are

$$
\begin{gathered}
\tau_{i j}=\mu\left(\frac{\partial u_{i}}{\partial x_{j}}+\frac{\partial u_{j}}{\partial x_{i}}-\frac{2}{3} \frac{\partial u_{k}}{\partial x_{k}} \delta_{i j}\right), \\
q_{i}=-\mu\left(\frac{C_{p}}{\operatorname{Pr}} \frac{\partial T}{\partial x_{i}}+\frac{Q}{S c} \frac{\partial Y}{\partial x_{i}}\right),
\end{gathered}
$$

where $\mu$ is the dynamic viscosity, and $\operatorname{Pr}$ and $S c$ are the Prandtl and Schmidt numbers, respectively. We take unity Lewis number $L e \equiv \operatorname{Pr} / S c=1$, with $\operatorname{Pr}=S c=0.75$. The dynamical viscosity is $\mu=1.7 \times 10^{-5} \mathrm{~N} \mathrm{~s} / \mathrm{m}^{2}$. The fuel-air mixture and burnt gas are assumed to be perfect gases with a constant molar mass $m=2.9 \times 10^{-2} \mathrm{~kg} / \mathrm{mol}$, with $C_{v}=5 R^{0} / 2 m, C_{p}=7 R^{0} / 2 m$, i.e., the adiabatic index $\gamma=C_{p} / C_{v}=1.4$, and the equation of state

$$
P=\rho R^{0} T / m,
$$

where $R^{0} \approx 8.31 \mathrm{~J} /(\mathrm{mol} \mathrm{K})$ is the universal gas constant. We consider a single-step irreversible Arrhenius reaction of the first order with an activation energy $E_{a}$ and a characteristic time $t_{R}$. In our simulations we took $E / R^{0} T_{u}=32$ in order to have better resolution of the reaction zone. The factor $t_{R}$ was adjusted to obtain a particular value of the unstretched laminar flame speed $S_{L}$ by solving the associated eigenvalue problem. We take the initial density, temperature, and pressure of the fuel mixture as $\rho_{u}=1.16 \mathrm{~kg} / \mathrm{m}^{3}, T_{u}=300 \mathrm{~K}$, and $P_{u}=10^{5} \mathrm{~Pa}$, respectively, with the thermal expansion ratio $\Theta=\rho_{u} / \rho_{b}=8$ and 14 . The flame thickness is conventionally defined as

$$
\delta_{L} \equiv \frac{\mu}{\operatorname{Pr} \rho_{u} S_{L}} .
$$

It is noted that $\delta_{L}$ is just a mathematical parameter of length dimension related to the flame front, while the effective flame thickness can appear considerably larger, see Ref. 36.

We take a rather large initial Mach number $M a=S_{L} / c_{s}=10^{-2}$ to achieve the saturation of the flame velocity at shorter length and time scales as compared to that in Ref. 28. Such a value of the Mach number may be attributed, for example, to hydrogen-oxygen flames. Besides, numerous computational studies of DDT employ even larger values of the Mach number, falling in the range $0.05-0.1$, in order to simplify the numerical task of DDT modeling, see, e.g., Refs. 33 and 37-39. 
We employ the moderate values of the channel width, $2 h=10 \delta_{L}-35 \delta_{L}$, which are comparable to the values used in previous studies. The choice of the channel width implies that the Reynolds numbers related to the planar flame velocity are in the range $R e=S_{L} h / v=h / \delta_{L} P r=6.67-23.33$. At the same time, the Reynolds number related to the flow, $R e_{f}=\left\langle u_{z}\right\rangle 2 h / v$, may be several orders of magnitude larger due to flame acceleration and thermal expansion of the burning gas.

We assume non-slip and adiabatic boundary conditions at the channel walls: $\mathbf{u}=0$ and $\mathbf{n} \cdot \nabla T=0$, where $\mathbf{n}$ is the unit normal vector at the walls. At the open end, non-reflecting boundary conditions are used. As initial conditions, we used planar flame "ignited" at the closed end of the channel, with the structure given by the analytical solution of Zel'dovich and Frank-Kamenetskii:2, 40

$$
\begin{gathered}
T=T_{u}+T_{u}(\Theta-1) \exp \left[-\left(z-z_{0}\right) / \delta_{L}\right], \text { if } z>z_{0}, \\
T=\Theta T_{u}, \text { if } z<z_{0}, \\
Y=\left(T_{b}-T\right) /\left(T_{b}-T_{u}\right), \quad P=P_{u}, \quad u_{x}=0, \quad u_{z}=0 .
\end{gathered}
$$

Here, $z_{0}$ denotes the initial flame position.

In our simulations, we used a 2D Cartesian hydrodynamic Navier-Stokes code adapted for parallel computation. ${ }^{41}$ We used mesh with variable resolution in order to take into account the growing distances between the channel end, the accelerating flame, and the shocks, and to resolve both chemical and hydrodynamic spatial scales. Typical computational resources for one such simulation required up to $10^{4} \mathrm{CPU}$-hours, which required the use of parallel calculations.

We used a rectangular grid with the grid walls parallel to the coordinate axes. The sketch of the calculation grid could be found in Refs. 28 and 33. The channel length exceeds the channel width significantly, reaching $10^{4} \mathrm{R}$ at the end of the simulation runs. To reduce the computational time, we made the grid spacing non-uniform along the z-axis with the zones of fine grid around the flame and leading shock fronts. In the flame and shock wave domains the grid size in the z-direction was, correspondingly, $0.25 \delta_{L}$ and $0.5 \delta_{L}$ for the main calculation runs, which allowed us to resolve the internal structure of the flame and shock waves. Outside the region of fine grid the mesh size grows gradually with $2 \%$ change in size between the neighboring cells. In order to keep the flame and shock waves in the zone of fine grid we implemented periodical mesh reconstruction during the calculation run. Splines of the third order are used for re-interpolation of the flow variables during periodic grid reconstruction to preserve second-order accuracy of the numerical scheme.

\section{SIMULATION RESULTS AND DISCUSSION}

A planar flame front ignited at the closed end was used as the initial condition, see Eqs. (22)(24), in order to focus on the critical expansion ratio phenomenon and CJ deflagrations, and to eliminate the influence of precursor acceleration, as will be discussed further.

Figure 3 shows the profiles of the scaled velocity evolution for $\Theta=8$ and the range of $R e=6.67-20.0$. Each velocity profile shows an intermediate (first saturation) stage with stationary velocity considerably below the $\mathrm{CJ}$ deflagration velocity, followed by additional acceleration and subsequent saturation to the $\mathrm{CJ}$ deflagration regime (second saturation). Each velocity graph is shown up to the instant of detonation onset; at certain flow parameters (e.g., for $R e=6.67$ ) the explosion and detonation start relatively fast. The flame accelerates according to the Shelkin mechanism until it reaches the first saturation stage. The additional acceleration and velocity rise after the first saturation stage are attributed to pre-heating of the fuel mixture due to viscous stress at the wall. As shown in Ref. 33, the effect of viscous heating is of lesser importance at the initial stages of flame acceleration (as low as $M a^{2} \ll 1$ ), although, this mechanism starts to dominate at the later stages. As a result, the temperature of the fuel mixture at the adiabatic wall becomes noticeably larger than that at the channel centerline. In particular, Fig. 4 shows (a) temperature and (b) velocity distributions across the channel centerline just ahead of the flame tip for different time instants $S_{L} t / h=2.61,7.65,10.43$, and 15.31 . The velocity profiles demonstrate extremely strong shear 


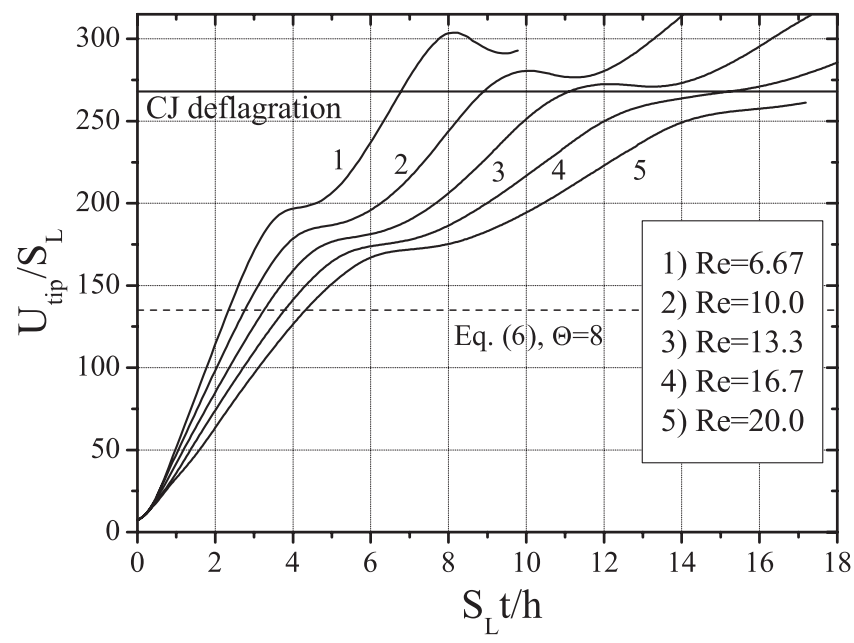

FIG. 3. Velocity evolution for $\Theta=8, R e=6.67-20.0$.

close to the walls, which is the source of viscous stress and heating. The strong velocity shear is one of the key elements in the Shelkin mechanism of flame acceleration, see the quantitative theory of the process in Refs. 18 and 19.

Figure 5 presents the temperature evolution at the channel centerline and at the wall (measured at a distance $h$ ahead of the flame tip) for $M a=0.01$ and $R e=13.3$. It is seen that the temperature at the channel centerline is in sync with the velocity evolution, while the temperature at the wall increases more monotonically. In order to demonstrate the effect of the role of the heating due to viscous stress more evidently, the temperature difference between the wall and the centerline is depicted versus the instantaneous flame tip Mach number in the laboratory frame in Fig. 6. Comparison of the numerical results and the theoretical predictions for the temperature at the channel centerline shows good agreement in spite of the simplifications adopted in the theory, thus supporting the isentropic approximation employed in the theoretical model. In agreement with the theoretical predictions, the heating due to viscous stress at the wall is minor before the flame reaches the first saturation stage (i.e., before Mach number reaches the value 1.8-1.9 in Fig. 6, corresponding to the normalized time instant $\sim 5-6 S_{L} t / h$ in Fig. 5); however, it continuously becomes prevailing afterwards. From Fig. 6, we observe a strong correlation between the onset of both saturation stages and the increasing role of viscous heating. At the same time, the instantaneous density drop and temperature jump at the flame front obtained from the numerical simulation indicate certain shortcomings of the present theoretical
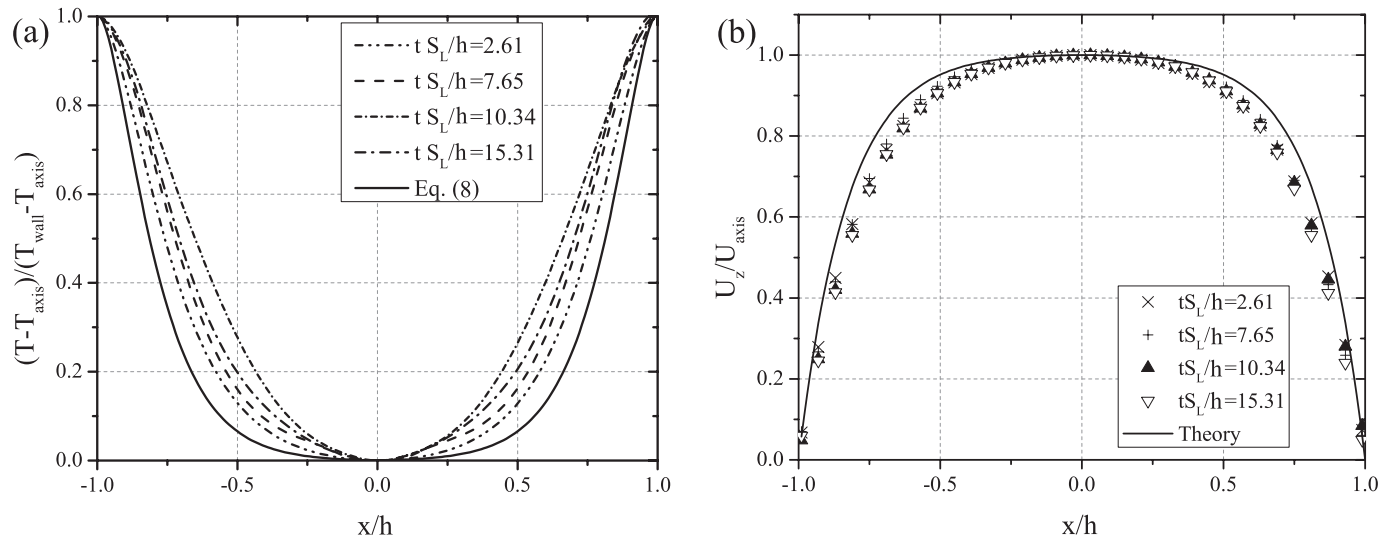

FIG. 4. (a) Temperature and (b) velocity profiles across the channel centerline ahead of the flame tip for time instants $S_{L} t / h=2.61,7.65,10.43$, and 15.31. The solid line in plot (b) is given by Eq. (8) of Ref. 18 . 


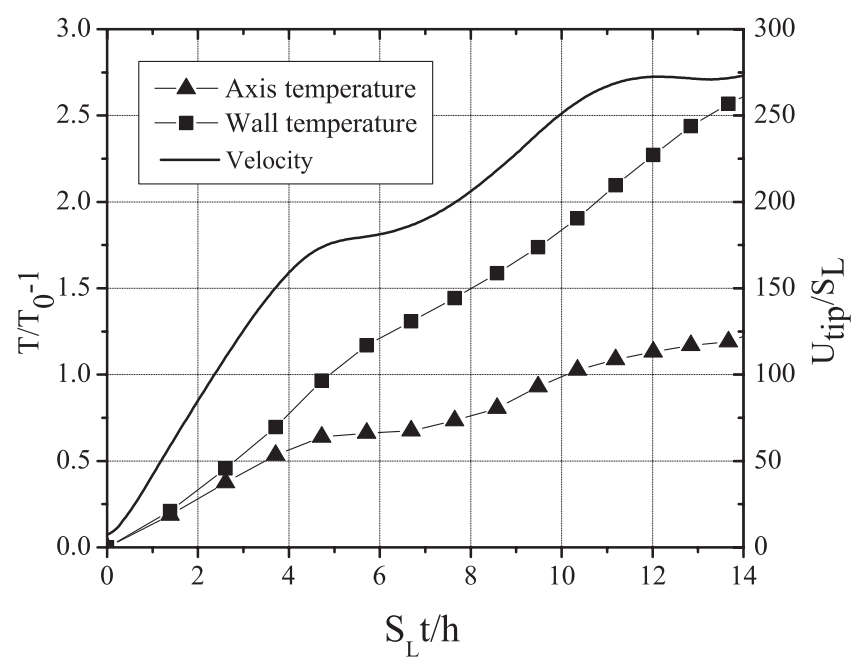

FIG. 5. Temperature evolution at the channel centerline and walls for $\Theta=8, \operatorname{Re}=13.3$.

model. In particular, Fig. 7 shows the density drop and temperature jump at the flame front to be about $\theta \approx 5$ at the time interval of intermediate quasi-saturation, $S_{L} t / h=5-7$. Though the attained value is much lower than the initial density drop $\Theta=8$, it is nevertheless noticeably larger than the critical value $\Theta_{C}=3$ predicted by the theory. As discussed in Sec. II, other physical mechanisms, e.g., hydraulic resistance, may lead to velocity saturation at higher values of the expansion ratio.

The flame speed at the second saturation regime is close to the physical limit of CJ deflagration speed, ${ }^{30,31}$ which is subsonic with respect to the fuel mixture ahead of the flame and supersonic in the laboratory frame. The turbulent high-speed flames observed experimentally and often referred to as "fast flames" 5,42 may be also associated with this regime. Figure 8 shows the first and second saturation velocity values for various Reynolds numbers for $\Theta=8$. The dependence of $M a_{w}$ on Re given by Eq. (14) for $\Theta=8$ is plotted in Fig. 8 by the dashed-dotted line. The first and second saturation velocities obtained in the numerical simulations are shown by triangles and squares, respectively. It is seen that the first (intermediate) saturation stage lies considerably lower than the CJ deflagration velocity, while the second saturation velocity is close to it for all Reynolds numbers. It is also observed that Eqs. (12)-(14) predict slightly lower first saturation velocity for lower $R e$

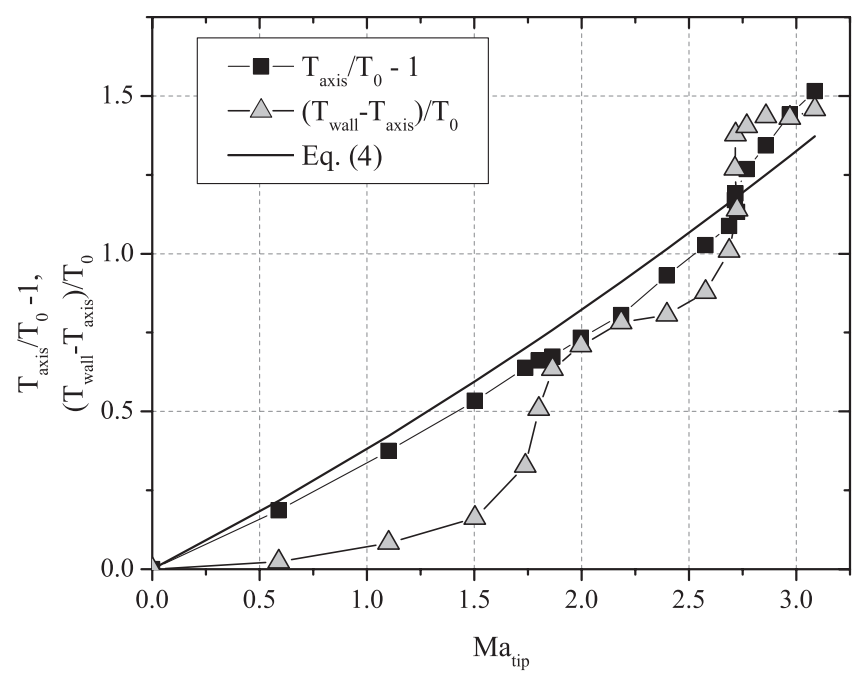

FIG. 6. Normalized temperature difference between the wall and the centerline versus the instantaneous flame tip Mach number, $R e=13.3$. 


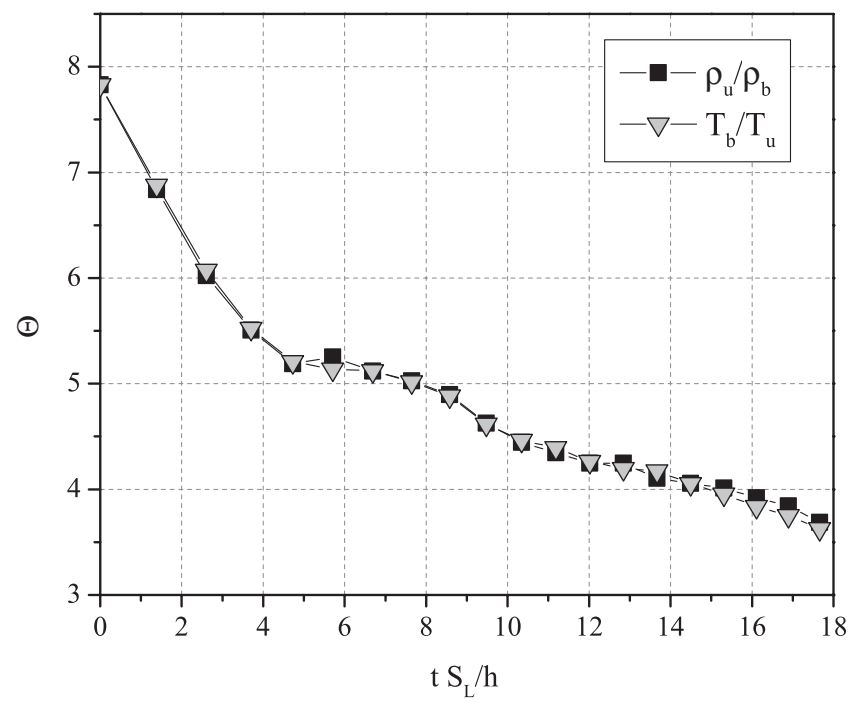

FIG. 7. Evolution of density and temperature drops at the flame front at the channel centerline, $R e=13.3$.

as compared to the simulation results. For sufficiently large values of the Reynolds number $R e$, the prediction of Eq. (14) lies between those of Eq. (6) $\left(U_{\text {sat,tip }} / S_{L} \approx 1.35\right)$ and the numerical results ( $U_{\text {sat,tip }} / S_{L} \approx 1.7$ for $R e=23.3$ ). It is noted that $S_{L}$ may be modified by increasing the mixture temperature, while Eqs. (6) and (12) do not account for the change of $S_{L}$ due to pre-heating. The second (final) saturation velocity approaches the CJ deflagration velocity with increasing channel width, which can be explained by the decreasing heating effect due to viscous stress in the boundary layer. It was recently shown that for shock waves and stable detonations in narrow channels the mean propagation velocity decreases for smaller channel widths due to dissipative effects at the walls. ${ }^{43,44}$

We point out that the recent experiments in channels with obstacles ${ }^{32}$ also show an intermediate stage of flame acceleration with quasi-stationary flame velocity similar to the present numerical modeling. The authors of Ref. 32 attributed this intermediate stationary velocity to interactions between leading shocks and flame. In addition, similar phenomenon was observed in the work on flame acceleration in obstructed channels ${ }^{45}$ for certain channel blockage ratios by carrying out the simulation long enough to obtain fast near-CJ deflagrations. It is also noted that the notion of critical expansion ratio was first introduced in the experimental work of Ref. 46, where it was shown that

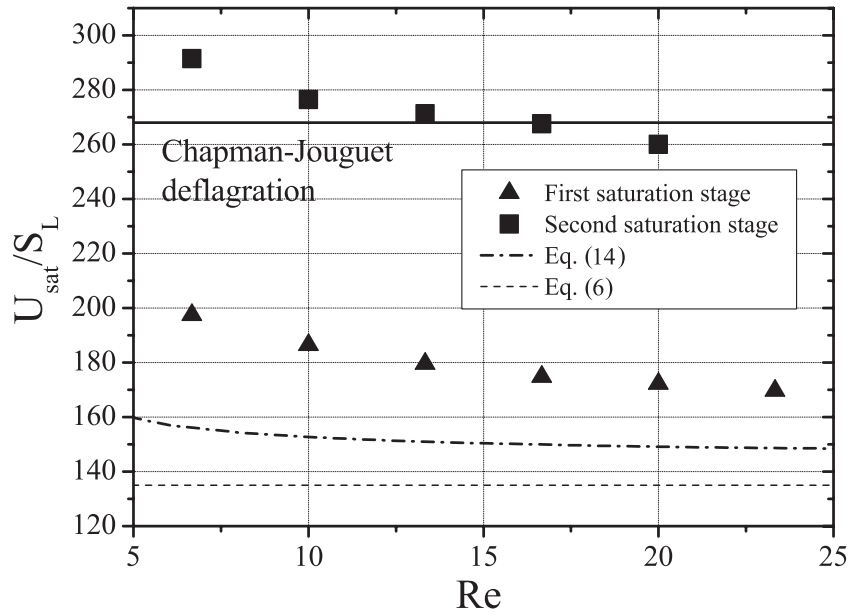

FIG. 8. Dependence of saturation velocities on $R e$ for the first and second stages, $\Theta=8$. 
the value of the thermal expansion ratio determines the possibility of flame acceleration to nearCJ deflagration speeds in channels with obstacles. For blockage ratios $0.3-0.6$ in the cylindrical tubes, the value of the critical thermal expansion ratio was experimentally found to lie in the range $\Theta_{C}=3.5-4$. It is interesting to note that this phenomenon is observed in the present simulations with smooth non-slip walls, based on a purely hydrodynamical mechanism developed in Refs. 18,19, and 34. Since the theory predicts the critical value $\Theta_{C}=3$ for $2 \mathrm{D}$ channels being 1.5 times larger than that for cylindrical tubes, $\Theta_{C}=2$, the experimental results of Ref. 46 can be interpreted as $\Theta_{C} \approx 5.5$ for $2 \mathrm{D}$ channels, being quite in line with the present numerical simulations. The concept of critical expansion ratio is also relevant to fuel-injection flashback safety of hydrogen-enriched fuels. In particular, it was recently shown that the effect of gas expansion is related to the flashback of premixed hydrogen-air flames in the boundary layer of a turbulent channel flow. ${ }^{47}$ The study of Ref. 47 focused mainly on the influence of the Darrieus-Landau instability $2,30,40,48$ on the flashback process, however, for preheated fuels the expansion ratio is close to the theoretical predictions for $\Theta_{C}$. Therefore, provided that preheating is low enough, the coupling of the non-slip boundary conditions and gas expansion would affect the upstream flame propagation during flashback.

We stress that while the intermediate saturation of the flame velocity below the CJ value is common, it is not a universal one. For example, our simulation for a higher initial value of the expansion factor, $\Theta=14$, did not show such an intermediate saturation as shown in Fig. 9. Here the first saturation stage is no longer distinctive, although for all Reynolds numbers, the velocity evolution passes a noticeable flex point near $M a_{\text {tip }}=1.27$. In Fig. 9, the flex point is related to a strong decrease of the instantaneous expansion ratio $\theta$ approaching the critical value $\Theta_{C}$ because of the gas compression.

On the other hand, the flex-point in evolution of the accelerating flame velocity may be caused by other physical mechanisms, which should be distinguished carefully. For example, Ref. 33 reported a flex-point at the velocity evolution due to transition from the precursor flame acceleration to the Shelkin acceleration mechanism as elucidated below. In experiments ignition is typically initiated as a hemi-spherical flame ball rather than a planar flame. This may lead to considerable modification of the flame velocity evolution at the very early stage of flame acceleration due to the so-called precursor (or "finger") flame acceleration. ${ }^{49,50}$ Here we emphasize that the precursor flame acceleration is short but powerful, being much stronger than the Shelkin acceleration during the period of its viability. To illustrate the modification of the velocity evolution due to precursor acceleration in channels with non-slip walls, the initial conditions in the forms of a flame ball and a planar front were compared in Figure 4(b) of Ref. 33. It was observed that the velocity evolution undergoes a noticeable flex point

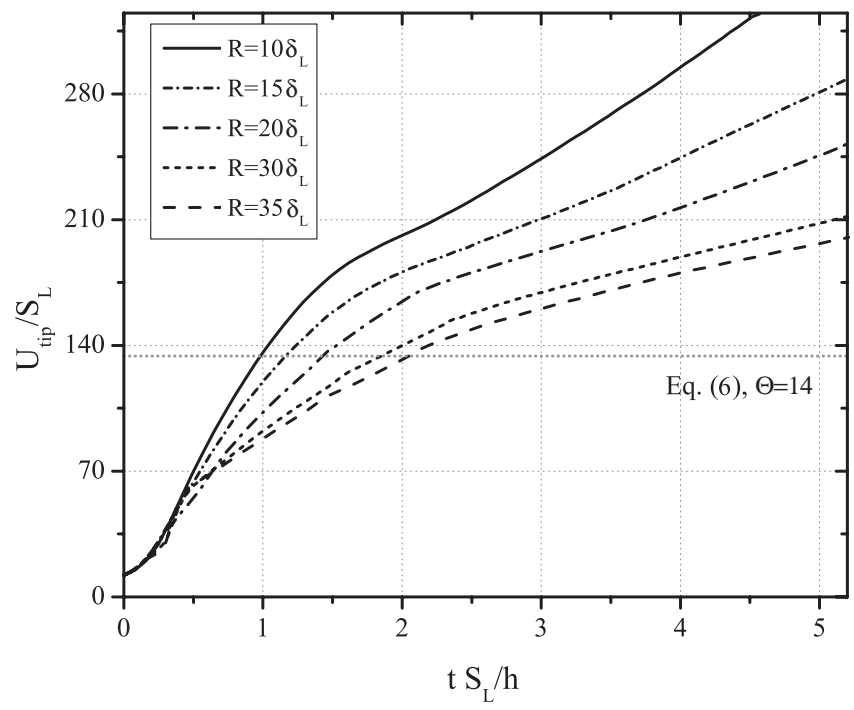

FIG. 9. Velocity evolution for $\Theta=14, h=10 \delta_{L}-35 \delta_{L}$. 
at a relatively early time, which resembles the saturation stages of Fig. 8. However, the nature of this flex point is substantially different from the effects studied in the present paper. In contrast with the CJ deflagration and velocity saturation due to attainment of the critical expansion ratio, the observed velocity jump is related to the precursor acceleration of finger-shaped flames. ${ }^{49,50}$ Unlike the late stages investigated in the present work, the precursor flame acceleration effect can be distinguished by the early time when it is observed. The normalized time of the local maximum of the flame velocity evolution in the precursor acceleration is ${ }^{50}$

$$
t_{\mathrm{wall}}=\frac{\ln \Theta}{\Theta-1},
$$

which corresponds to the time when the skirt of the elongated flame touches the channel wall, and is almost independent of the initial Mach number. ${ }^{50}$ For $\Theta=8$, the normalized time in planar geometry predicted by Eq. (25) is $t_{\text {wall }} \approx 0.29$. The normalized maximum velocity can be quite high for high $\Theta$, being equal to $U_{\max } / S_{L}=\Theta^{2}$ in the low-Mach number approximation. However, compressible theory and simulations of Ref. 50 considerably modified this result, predicting a lower value of $U_{\max } / S_{L}$ for $M_{0}=0.04$ as compared to low Mach numbers.

Finally, we discuss the development of explosion and detonation onset at the late stages of flame acceleration. The flow remains laminar in the entire acceleration process, but at later times explosion of the fuel mixture starts gradually with the signs of flow turbulization due to instabilities similar to those discussed in Ref. 51 for $M a=10^{-3}$. The flow becomes more complicated during explosion and detonation triggering. We observe that in the case of realistic initial flame Mach number $\left(M a=10^{-2}\right)$, explosion of the preheated gas at the walls starts rather slowly, without direct formation of the detonation wave, as compared to, e.g., Ref. 37. The entire multi-dimensional picture of the final stage of the DDT is presented in Fig. 10, which shows all elements of the flame dynamics at that stage. The first snapshot shows the elongated flame front at the very beginning of the explosion. It is

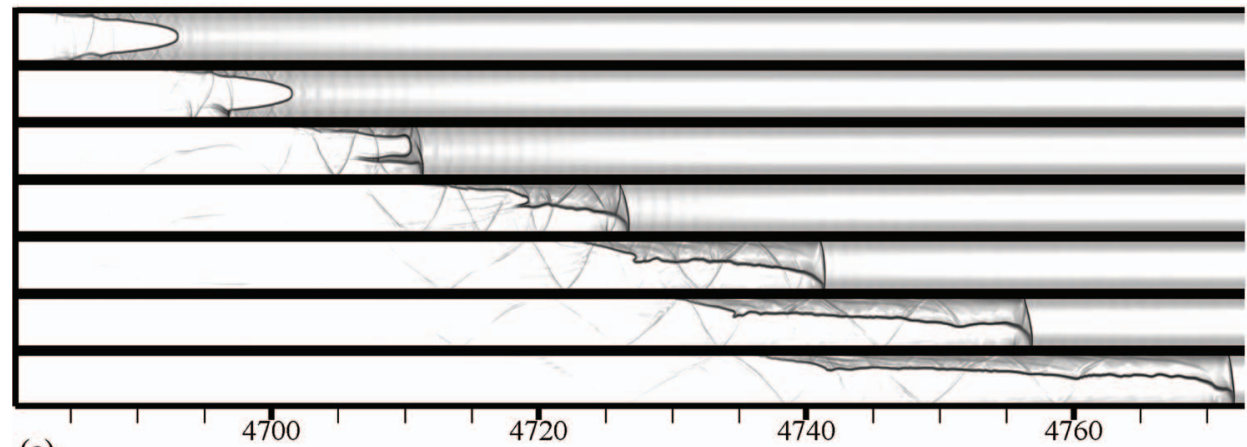

(a)

$$
\mathrm{Z} / \mathrm{h}
$$

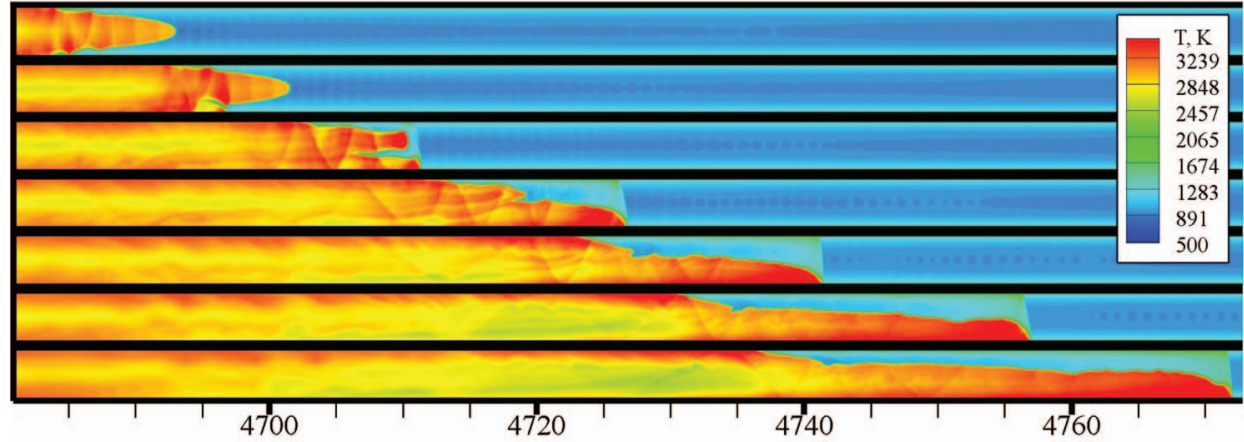

(b)

$$
\mathrm{Z} / \mathrm{h}
$$

FIG. 10. (a) Numerical schlieren (based on density gradient) and (b) temperature field during deflagration-to-detonation transition for planar geometry, $M a=0.01, R e=13.3\left(h=20.0 \delta_{L}\right)$. $\mathrm{Z}$ to $\mathrm{X}$ aspect ratio is 0.5 . Time instants are equally spaced in the range (19.2-19.41) $S_{L} t / h$. 
seen that the explosion starts at the wall in agreement with the theory and simulations of Ref. 33 . The process is more pronounced in the second snapshot. Tongue of the explosion bursts along the wall at high speed (second snapshot), catches up with the flame tip (third snapshot), and then leaves it far behind engulfing the flame (fourth snapshot and on). The burning region at the stage of explosion resembles the characteristic shape of an accelerating turbulent flame observed in experiments. ${ }^{5,14,15}$ We point out a strongly asymmetric explosion development, which differs noticeably from the symmetric DDT process described in Ref. 51. Still, at present we do not see any physical process responsible for losing or, respectively, keeping symmetry in DDT. Presumably, the choice between symmetrical or asymmetrical explosion development is purely stochastic, related to instabilities and turbulence arising in the DDT. Experimental studies typically describe the explosion process as spreading of fast turbulent burning in a boundary layer. The last snapshot of Fig. 10 depicts the system, which is not yet detonation, but is already the complex of supersonic flame and a shock wave. It is seen from Fig. 10 that, as compared to the case of initial Mach number $M a=0.001$ reported in Ref. 51, the explosion has similar duration in terms of $S_{L} t / h$ but is essentially non-symmetrical, in that sense similar to that observed in Ref. 52.

Consequently, the process of flame acceleration studied herein is universal from the hydrodynamic point of view, and it is adequately described even by using the simplified one-step Arrhenius kinetics. In contrast, the process of explosion and detonation triggering is highly sensitive to the details of the chemical kinetics, which has to include low-temperature reactions together with hightemperature mechanism. For this reason, Fig. 10 can be considered as a qualitative illustration instead of a qualitative analysis of the physical mechanism of the explosion.

\section{SUMMARY}

In this study, we have shown that the velocity evolution in the process of premixed flame acceleration in narrow channels with non-slip adiabatic walls may undergo two quasi-stationary stages: an intermediate stage with velocity noticeably below the CJ deflagration speed, to be followed by additional flame acceleration, and a subsequent regime in which the flame velocity saturates to the CJ deflagration. The existence of the intermediate quasi-steady stage is explained by the combined effect of gas compression ahead of the flame front and the hydraulic resistance. The theoretical analysis of the present study is based on the concept of critical expansion ratio: the acceleration rate strongly depends on the thermal expansion ratio at the flame front, ${ }^{18,19}$ with the acceleration process ceasing completely for expansion ratios below a certain critical value $\Theta_{C}$. We estimate the first quasi-steady saturation velocity of the flame tip in the laboratory frame with and without heating due to viscous stress and compare the theoretical estimates with the numerical results. Numerical simulations show that, in agreement with the theoretical predictions, heating due to viscous stress at the wall is minor before the flame reaches the first quasi-steady stage and prevails afterwards. It is demonstrated that the additional acceleration is related to viscous heating at the channel wall. The deflagration-to-detonation transition and the development of explosion at the late stages of flame acceleration is discussed qualitatively as well.

\section{ACKNOWLEDGMENTS}

The work at Umeå University was supported by the Swedish Research Council (VR), the Kempe Foundation, and Stiftelsen Lars Hiertas Minne Grant No. FO2010-1015. Numerical simulations were performed at High Performance Computer Center North (HPC2N), Umeå, Sweden, within SNAC Project No. 001-10-159. The work at Princeton University was supported by the U.S. Air Force Office of Scientific Research.

\section{APPENDIX: CONVERGENCE AND TEMPERATURE-DEPENDENT VISCOSITY TESTS}

In order to check if the resolution is sufficient to study the flame acceleration process, we performed resolution tests for $R e=6.67$. The grid size in flame domain varied between $0.125 \delta_{L}$, $0.25 \delta_{L}, 0.5 \delta_{L}$, and $1 \delta_{L}$. We checked the velocity of the flame tip at the various stages of flame velocity 
TABLE I. Resolution tests for $R e=6.67$.

\begin{tabular}{lcccccc}
\hline \hline$\Delta z_{f} / \delta_{L}$ & $U_{S 1} / S_{L}$ & $\Delta U_{S 1} / S_{L}$ & $U_{S 2} / \delta_{L}$ & $\Delta U_{S 2} / \delta_{L}$ & $t_{\mathrm{DDT}} S_{L} / h$ & $\Delta t_{\mathrm{DDT}} S_{L} / h$ \\
\hline 1.0 & 215.1 & & $\mathrm{~N} / \mathrm{A}$ & & 10.38 & \\
0.5 & 201.3 & 13.8 & 273.8 & & 10.1 & 0.28 \\
0.25 & 198.3 & 3.0 & 291.6 & 17.8 & 9.82 & 0.28 \\
0.125 & 197.8 & 0.5 & 306.4 & 14.8 & 10.0 & 0.18 \\
\hline \hline
\end{tabular}

saturation. The resolution test results are presented in Table I, which shows good convergence of the numerical solution at the flame saturation stage. Resolution tests also showed the tendency for convergence for the instant of the onset of DDT with increasing resolution. It is noted that convergence tests for DDT is a matter of controversy, ${ }^{39}$ with either the suggestion that it is not necessary due to the stochastic nature of the phenomenon, or performing them only for the deflagration stage. However, integrated values of interest, such as saturation velocities or pre-detonation times and distances, should demonstrate convergence to be considered reliable. ${ }^{39}$

Notation: $\Delta z_{f} / \delta_{L}$ is the spatial step in the flame grid domain; $U_{S 1} / S_{L}$ and $\Delta U_{S 2} / \delta_{L}$ are the flame saturation velocities at the first and second steps, respectively (see Fig. 8); $t_{\mathrm{DDT}} S_{L} / h$ is the scaled instant for the onset of DDT. $\Delta U_{S 1} / S_{L}$ and $\Delta U_{S 2} / \delta_{L}$ are the increments of $\Delta U_{S 1} / S_{L}$ and $U_{S 2} / \delta_{L}$ calculated in the table row $\mathrm{i}$ as $\Delta U_{S 1}(i)=U_{S 1}(i)-U_{S 1}(i-1)$ and $\Delta U_{S 2}(i)=U_{S 2}(i)-U_{S 2}(i-1)$. The increment for $t_{\mathrm{DDT}} S_{L} / h$ is calculated in a similar manner. Resolution in the wave grid domain is equal to $\Delta z_{w}=2 \times \Delta z_{f}$ for each run.

Similar to the majority of previous works on the subject, ${ }^{28,33,45,50}$ in the present paper we consider a constant viscosity coefficient independent of temperature. In order to substantiate the use of the constant viscosity, we performed additional test simulations for the temperature-dependent viscosity $\mu(T)=\mu_{0}\left(T / T_{u}\right)^{0.7}$, with constants $\mu_{0}=\mu$ and $T_{u}$ defined in Sec. III. We kept the same value of laminar burning velocity $S_{L}$ by solving the associated eigenvalue problem for the temperaturedependent viscosity. It was found that the flame velocity evolution for the temperature-dependent viscosity is qualitatively identical to that for the constant viscosity, exhibiting two quasi-steady saturation stages and thus justifying the main result of the present study. It was also found that the thermal flame thickness becomes larger for the case of temperature-dependent viscosity, with the overall flame structure being qualitatively similar.

${ }^{1}$ K. I. Shelkin, "Influence of tube roughness on the formation and detonation propagation in gas," Zh. Eksp. Teor. Fiz. 10, 823 (1940).

${ }^{2}$ Ya. B. Zeldovich, G. I. Barenblatt, V. B. Librovich, and G. M. Makhviladze, Mathematical Theory of Combustion and Explosion (Consultants Bureau, New York, 1985).

${ }^{3}$ J. E. Shepherd and J. H. S. Lee, Major Research Topics in Combustion (Springer-Verlag, Hampton, 1992).

${ }^{4}$ G. D. Roy, S. M. Frolov, A. A. Borisov, and D. W. Netzer, "Pulse detonation propulsion: challenges, current status, and future perspective," Prog. Energy Combust. Sci. 30, 545 (2004).

${ }^{5}$ G. Ciccarelli and S. Dorofeev, "Flame acceleration and transition to detonation in ducts," Prog. Energy Combust. Sci. 34, 499 (2008).

${ }^{6}$ S. B. Dorofeev, "Flame acceleration and explosion safety applications," Proc. Combust. Inst. 33, 2161-2175 (2011).

${ }^{7}$ A. Y. Poludnenko, T. A. Gardiner, and E. S. Oran, "Spontaneous transition of turbulent flames to detonations in unconfined media," Phys. Rev. Lett. 107(5), 054501 (2011).

${ }^{8}$ V. Bychkov, D. Valiev, and L.-E. Eriksson, "Physical mechanism of ultrafast flame acceleration," Phys. Rev. Lett. 101, 164501 (2008).

${ }^{9}$ E. Oran and V. Gamezo, "Origins of the deflagration-to-detonation transition in gas-phase combustion," Combust. Flame 148, 4-47 (2007).

${ }^{10}$ Y. Gao and C. K. Law, "Detonative propagation and accelerative expansion of the Crab Nebula shock front," Phys. Rev. Lett. 107, 171102 (2011).

${ }^{11}$ V. Bychkov, P. Matyba, V. Akkerman, M. Modestov, D. Valiev, C. K. Law, G. Brodin, M. Marklund, and L. Edman, "Speedup of doping fronts in organic semiconductors through plasma instability," Phys. Rev. Lett. 107, 016103 (2011).

${ }^{12}$ M. Modestov, V. Bychkov, and M. Marklund, "Ultrafast spin avalanches in crystals of nanomagnets in terms of magnetic detonation,” Phys. Rev. Lett. 107, 207208 (2011).

${ }^{13}$ C. Dion, O. Jukimenko, M. Modestov, M. Marklund, and V. Bychkov, "Anisotropic properties of spin avalanches in crystals of nanomagnets," Phys. Rev. B 87, 014409 (2013).

${ }^{14}$ P. A. Urtiew and A. K. Oppenheim, "Experimental observations of the transition to detonation in an explosive gas," Proc. R. Soc. London, Ser. A 295, 13 (1966). 
${ }^{15}$ M. Kuznetsov, V. Alekseev, I. Matsukov, and S. Dorofeev, "DDT in a smooth tube filled with a hydrogen-oxygen mixture," Shock Waves 14, 205 (2005).

${ }^{16}$ D. J. Finigan, B. D. Dohm, J. A. Mockelman, and M. A. Oehlschlaeger, "Deflagration-to-detonation transition via the distributed photo ignition of carbon nanotubes suspended in fuel/oxidizer mixtures," Combust. Flame 159, 1314-1320 (2012).

${ }^{17}$ Y. Huang, H. Tang, J. Li, and C. Zhang, "Studies of DDT enhancement approaches for kerosene-fueled small-scale pulse detonation engines applications," Shock Waves 22(6), 615-625 (2012).

${ }^{18}$ V. Bychkov, A. Petchenko, V. Akkerman, and L.-E. Eriksson, "Theory and modeling of accelerating flames in tubes," Phys. Rev. E 72, 046307 (2005).

${ }^{19}$ V. Akkerman, V. Bychkov, A. Petchenko, and L.-E. Eriksson, "Accelerating flames in cylindrical tubes with nonslip at the walls," Combust. Flame 145(1-2), 206-219 (2006).

${ }^{20}$ I. Brailovsky and G. I. Sivashinsky, "Hydraulic resistance as a mechanism for deflagration-to-detonation transition," Combust. Flame 122, 492-499 (2000).

${ }^{21}$ I. Brailovsky, L. Kagan, and G. I. Sivashinsky, “Combustion waves in hydraulically resisted systems," Philos. Trans. R. Soc. London, Ser. A 370, 625-646 (2012).

${ }^{22}$ V. S. Babkin, "Fast gas combustion in systems with hydraulic resistance," Combust., Explos. Shock Waves 48, 278-287 (2012).

${ }^{23}$ V. N. Kurdyumov and M. Matalon, "Flame acceleration in long narrow open channels," Proc. Combust. Inst. 34, 865-872 (2013).

${ }^{24} \mathrm{M}$. Wu, M. Burke, S. Son, and R. Yetter, "Flame acceleration and the transition to detonation of stoichiometric ethylene/oxygen in microscale tubes," Proc. Combust. Inst. 31, 2429 (2007).

${ }^{25}$ M.-H. Wu and C.-Y. Wang, "Reaction propagation modes in millimeter-scale tubes for ethylene/oxygen mixtures," Proc. Combust. Inst. 33, 2287 (2011).

${ }^{26}$ M.-H. Wu and W.-C. Kuo, "Transition to detonation of an expanding flame ring in a sub-millimeter gap," Combust. Flame 159, 1366-1368 (2012).

${ }^{27}$ M.-H. Wu and W.-C. Kuo, "Accelerative expansion and DDT of stoichiometric ethylene/oxygen flame rings in micro-gaps," Proc. Combust. Inst. 34, 2017-2024 (2013).

${ }^{28}$ D. Valiev, V. Bychkov, V. Akkerman, and L.-E. Eriksson, "Different stages of flame acceleration from slow burning to Chapman-Jouguet deflagration," Phys. Rev. E 80, 036317 (2009).

${ }^{29}$ V. Bychkov, V. Akkerman, D. Valiev, and C. K. Law, "Role of compressibility in moderating flame acceleration in tubes," Phys. Rev. E 81, 026309 (2010).

${ }^{30}$ L. D. Landau and E. M. Lifshitz, Fluid Mechanics (Pergamon Press, Oxford, 1989).

${ }^{31}$ R. Chue, J. Clarke, and J. H. Lee, “Chapman-Jouguet deflagrations,” Proc. R. Soc. London, Ser. A 441, 607 (1993).

${ }^{32}$ C. Johansen and G. Ciccarelli, "Visualization of the unburned gas flow field ahead of an accelerating flame in an obstructed square channel," Combust. Flame 156, 405-416 (2009).

${ }^{33}$ D. Valiev, V. Bychkov, V. Akkerman, and L.-E. Eriksson, "Heating of the fuel mixture due to viscous stress ahead of accelerating flames in deflagration-to-detonation transition," Phys. Lett. A 372, 4850 (2008).

${ }^{34}$ V. Akkerman, C. K. Law, V. Bychkov, and L.-E. Eriksson, "Analysis of flame acceleration induced by wall friction in open tubes," Phys. Fluids 22, 053606 (2010).

${ }^{35}$ V. Bychkov and V. Akkerman, "Explosion triggering by an accelerating flame," Phys. Rev. E 73, 066305 (2006).

${ }^{36}$ V. Akkerman, V. Bychkov, A. Petchenko, and L.-E. Eriksson, "Flame oscillations in tubes with nonslip at the walls," Combust. Flame 145, 675-687 (2006).

${ }^{37}$ L. Kagan and G. Sivashinsky, "The transition from deflagration to detonation in thin channels," Combust. Flame 134, 389 (2003).

${ }^{38}$ L. Kagan, "On the transition from deflagration to detonation in narrow channels," Math. Model. Nat. Phenom. 2, 40-55 (2007).

${ }^{39}$ L. Kagan and G. Sivashinsky, "On the transition from deflagration to detonation in narrow tubes," Flow, Turbul. Combust. 84, 423-437 (2010).

${ }^{40}$ C. K. Law, Combustion Physics (Cambridge University Press, New York, 2006).

${ }^{41}$ C. Wollblad, L. Davidson, and L.-E. Eriksson, "Large eddy simulation of transonic flow with shock wave/turbulent boundary layer interaction," AIAA J. 44, 2340 (2006).

${ }^{42}$ M. Kuznetsov, V. Alekseev, Yu. Yankin, and S. Dorofeev, "Slow and fast deflagrations in hydrocarbon-air mixtures," Combust. Sci. Technol. 174, 157-172 (2002).

${ }^{43}$ A. Chinnayya, A. Hadjadj, and D. Ngomo, "Computational study of detonation wave propagation in narrow channels," Phys. Fluids 25, 036101 (2013).

${ }^{44}$ D. Ngomo, A. Chaudhuri, A. Chinnayya, and A. Hadjadj, "Numerical study of shock propagation and attenuation in narrow tubes including friction and heat losses," Comput. Fluids 39, 1711-1721 (2010).

${ }^{45}$ D. Valiev, V. Bychkov, V. Akkerman, C. K. Law, and L.-E. Eriksson, "Flame acceleration in channels with obstacles in the deflagration-to-detonation transition," Combust. Flame 157, 1012-1021 (2010).

${ }^{46}$ S. B. Dorofeev, M. S. Kuznetsov, V. I. Alekseev, A. A. Efimenko, and W. Breitung, "Evaluation of limits for effective flame acceleration in hydrogen mixtures," J. Loss Prev. Process Ind. 14, 583-589 (2001).

${ }^{47}$ A. Gruber, J. H. Chen, D. Valiev, and C. K. Law, "Direct numerical simulation of premixed flame boundary layer flashback in turbulent channel flow," J. Fluid Mech. 709, 516-542 (2012).

${ }^{48}$ M. Modestov, V. Bychkov, D. Valiev, and M. Marklund, "Growth rate and the cutoff wavelength of the Darrieus-Landau instability in laser ablation," Phys. Rev. E 80, 046403 (2009).

${ }^{49}$ V. Bychkov, V. Akkerman, G. Fru, A. Petchenko, and L.-E. Eriksson, "Flame acceleration in the early stages of burning in tubes," Combust. Flame 150, 263-276 (2007). 
${ }^{50}$ D. Valiev, V. Akkerman, M. Kuznetsov, L.-E. Eriksson, C. K. Law, and V. Bychkov, "Influence of gas compression on flame acceleration in the early stage of burning in tubes," Combust. Flame 160(1), 97-111 (2013).

${ }^{51}$ V. Bychkov, D. Valiev, V. Akkerman, and C. K. Law, "Gas compression moderates flame acceleration in deflagration-todetonation transition," Combust. Sci. Technol. 184, 1066-1079 (2012).

${ }^{52}$ A. V. Gaathaug, K. Vaagsaether, and D. Bjerketvedt, "Experimental and numerical investigation of DDT in hydrogen-air behind a single obstacle,” Int. J. Hydrogen Energy 37(22), 17606-17615 (2012). 